\title{
Bending Process Analysis and Structure Design of Orthodontic Archwire Bending Robot
}

\author{
Jiang Jin-gang*, Zhang Yong-de, Jin Ming-liang and Wei chun-ge \\ Intelligent Machine Institute, Harbin University of Science and Technology, \\ Heilongjiang Harbin 150080, China \\ *jiangjingang1982@163.com
}

\begin{abstract}
Archwire bending is conducted by orthodontist in orthodontic treatment. However, this procedure is boring and time-consuming. In order to reduce the dependence on human technology in archwire bending forming, decrease the labor intensity of dentists and technicians, and improve the degree of automation of the orthodontic process. This paper proposes to use robots to replace dentists for completing orthodontic wires bending. Quantitative model of orthodontic wires with canine eminace is established based on Power function model. On the basis of bending process analysis of orthodontic wires, the overall structure, bend die and archwire supporting part of archwire bending robot for orthodontic treatment is designed.
\end{abstract}

Keywords: orthodontic archwire bending robot; bending process analysis; structure design

\section{Introduction}

In human growth process, malocclusion is a common kind of mouth disease due to genetic and environmental factors. It not only affects appearance or the growth of maxillofacial but also reduces masticatory function. With the development of the society, more and more people begin to pay attention to dental health and aesthetics. Wearing orthodontic wires is the most common and effective method for orthodontic treatment [1]. Traditionally, formed orthodontic wires are bended by doctor or technician manually. Manual method needs revising many times which leads to a waste of time and the increase medical expenses per patient. Furthermore, the accuracy is low and manipulator is always very tired after bending a few of orthodontic wires [2, 3]. It will greatly improve efficiency if use robot to finish this job, and it will decrease the time of bending an orthodontic wire. And the most important thing is that medical expenses will be decreased tremendously.

Some studies have been performed in the area of orthodontics and prosthodontics using robot. Robot-assisted therapy system with the function of producing x-ray images of teeth is invented to help on more accurately diagnosing dental erosion and bone loss [4]. Chewing robot with human skull mode is made to help on quantitatively studying human mastication and its motion pattern [5]. Oral rehabilitation robot with 6-DOF is manufactured for mouth opening and closing training [6]. Jaw movement training robot is built for intermaxillary traction therapy [7]. Some studies on computer-aided surgery with modular software system are applied on denture prosthodontics cultivation [8]. A new method for 3-D orthodontic treatment simulation was developed for an orthodontic planning system and an original system for three-dimensional reconstruction of dental anatomy was developed [9]. A threedimensional image software used in orthodontics and prosthodontics treatment was 
introduced by the SURESMILE company. And the company also presented to use manipulator to bend orthodontic wires [10].

This paper focuses on the orthodontic archwire bending robot. Quantitative model of orthodontic wires with canine eminace is established based on Power function model. On the basis of bending process analysis of orthodontic wires, archwire bending using robot is proposed. The overall structure, bend die and archwire supporting part of archwire bending robot for orthodontic treatment is designed.

\section{Quantitative Model of Orthodontic Wires with Canine Eminace}

For a long time, most of the researchers in the world study on orthodontic wires qualitatively. And its development greatly depends on the experience of doctors. This traditional qualitative way could not describe the process of the doctor's work. However, this kind of method hampers the development of orthodontics in the modern digital world. In order to realize digital and quantification, many scholars have studied it in depth at home and abroad. Power function model and Beta function model are the two kinds of model which are exist, and power function model is situable $[11,12]$. So in this paper we chose power function model to describe orthodontic wires curve with canine eminace. Figure 1 shows the formed wires with canine eminace.

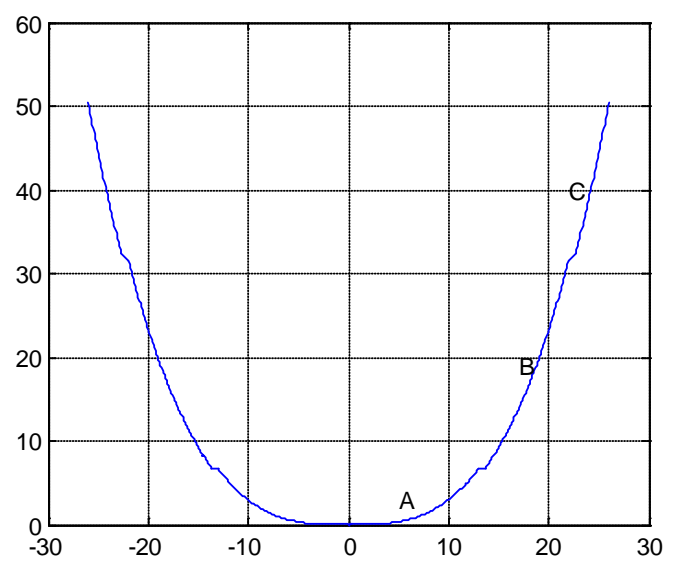

Figure 1. Formed Archwire with Canine Eminace

From Figure 1 we can see that the wire is basically symmetric, so we only have to study half of it. The right-hand wire is divided into three parts which are A, B and C by canine eminace. Canine eminace can be regarded as straight line because the distance of canine eminace are all very small. In this paper, we use Equation 1 to describe part $\mathrm{A}$. And then we get part $\mathrm{B}$ and part $\mathrm{C}$ by extending out part $\mathrm{A}$.

$$
y=\alpha x^{\beta} \quad x \geq 0
$$

Where $\alpha$ and $\beta$ are characteristic parameters of formed wire which can be fitted by Equation 2.

$$
\left\{\begin{array}{l}
\beta=\sigma(S / W-\mu L / W)^{\tau} \\
\alpha=L / W^{\beta}
\end{array}\right.
$$


Where $S, W, L$ represent the arc length, bow width and bow length of the half orthodontic wire respectively. $\sigma, \mu$ and $\tau$ are empirical fit constant. $\sigma=10.889, \mu=0.88$ and $\tau=3$. In order to analyze easily, change Equation 1 into parametric equation which is given as Equation 3 .

$$
C_{A}(t)=[x(t), y(t)] \quad t \in[0, W / 2]
$$

Then we can describe arc B using Eq.4 and arc C's equation is shown in Equation 5.

$$
\begin{array}{ll}
C_{B}(t)=C_{A}(t)+N_{A}(t) \cdot d_{1} & t \in[0, W / 2] \\
C_{C}(t)=C_{A}(t)+N_{A}(t) \cdot d_{2} & t \in[0, W / 2]
\end{array}
$$

Where $N_{A}(t)$ is the unit normal vector at a point of the orthodontic wire curve. Now we can describe the right-hand part wire using a piecewise function which is shown in Equation 6.

$$
\begin{cases}\left\{\begin{array}{c}
x=t \\
y=\alpha \cdot t^{\beta}
\end{array}\right. & t \in\left[0, t_{1}\right] \\
\left\{\begin{array}{c}
x=t+\frac{d_{1} \cdot \alpha \cdot \beta \cdot t^{\beta-1}}{\sqrt{1+\alpha^{2} \cdot \beta^{2} \cdot t^{2 \beta-2}}} \\
y=\alpha \cdot t^{\beta}-\frac{d_{1}}{\sqrt{1+\alpha^{2} \cdot \beta^{2} \cdot t^{2 \beta-2}}}
\end{array}\right. & t \in\left[t_{2}, t_{3}\right] \\
\left\{\begin{array}{l}
x=t+\frac{d_{2} \cdot \alpha \cdot \beta \cdot t^{\beta-1}}{\sqrt{1+\alpha^{2} \cdot \beta^{2} \cdot t^{2 \beta-2}}} \\
y=\alpha \cdot t^{\beta}-\frac{d_{2}}{\sqrt{1+\alpha^{2} \cdot \beta^{2} \cdot t^{2 \beta-2}}}
\end{array}\right. & t \in\left[t_{4}, t_{5}\right] \\
\left\{\begin{array}{c}
x=t \\
y=y_{1}+\frac{\left(y_{2}-y_{1}\right)\left(x-x_{1}\right)}{\left(x_{2}-x_{1}\right)}
\end{array}\right. & t \in\left[x_{1}, x_{2}\right] \\
\left\{\begin{array}{c}
x=t \\
y=y_{3}+\frac{\left(y_{4}-y_{3}\right)\left(x-x_{3}\right)}{\left(x_{4}-x_{3}\right)}
\end{array}\right. & t \in\left[x_{3}, x_{4}\right]\end{cases}
$$

Where $t$ is the abscissa axis of a point of orthodontic wire curve. $t_{1}$ and $t_{3}$ are the initial point coordinate of canine eminace while $t_{2}$ and $t_{4}$ are the termination point coordinate.$d_{1}$ and $d_{2}$ are the distances that arc A will be extended to form arc B and C. $x_{1}$ is the abscissa axis of arc A's terminal point, $x_{2}$ is the abscissa axis of arc B's origin point, $x_{3}$ is the abscissa axis of arc B's terminal point, $x_{4}$ is the abscissa axis of arc C's origin point. So we will get the orthodontic wire curve as long as we have the parameter $t_{1}, t_{3}, d_{1}$ and $d_{2}$.

\section{Bending Process Analysis of Orthodontic Wires}

Orthodontic wire in edgewise appliance includes conventional sequence bending and treatment of close loop. The common sequence of bending in the dentition model in general is between the canine and lateral incisor and first molar and the second molar, and the position 
is fixed. Treatment for close loop is the need to increase the gap or reduce the gap in the appropriate location, so the location is not fixed. Orthodontic archwire bending with close loop is as shown in Figure 2. As can be seen from the Figure 2, canine eminace bend and molars eminace are curved in the horizontal direction, and close loop is in the vertical direction for bending.

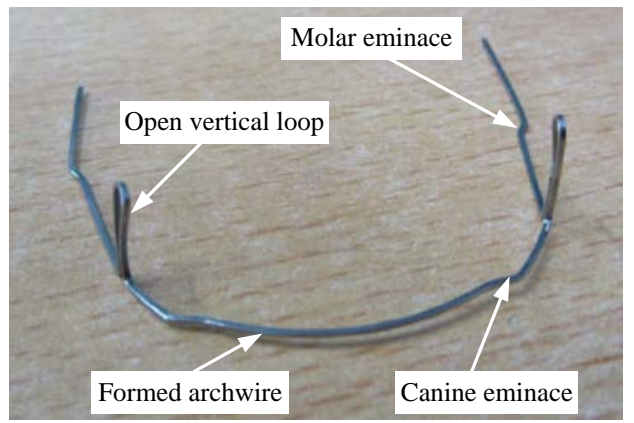

Figure 2. Formed Archwire with Close Loop

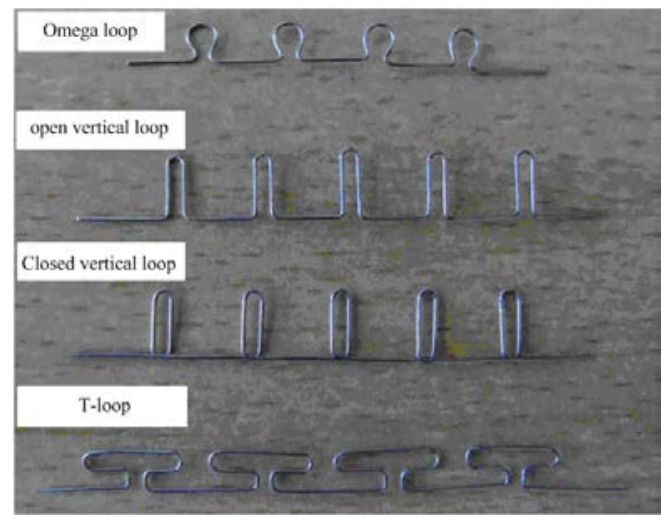

Figure 3. Close Loop Form

In the process of orthodontic archwire bending, the close loop as a strength unit is mainly used for aligning the teeth and closing extraction space. It needs to bend into different shapes according to malocclusion patients. Figure 3 is common shape used in close loop. Because of its complex shape and diversity, the bending forming is very difficult. And the process is directly related to the efficiency and quality of forming. Because the outside close loop parts can be bended by each control node corresponding to the angle of the bending forming, so this paper only analyzes the bending process scheme of close loop.

\subsection{Bending Process Scheme of Open Vertical Loop}

In the orthodontic archwire bending process, the bending die driven by archwire bending motor rotates in accordance with its own rotary center. If you want to make the mold rotating in the opposite direction to realize archwire bending work, you must make fine adjustments of opponent gripper position in the Y direction so that the rotary center of the bending mold is repositioned. The action must also relate to the movement in the $\mathrm{Z}$ direction, in order to avoid interference. And the same function can be achieved by the rotation of archwire, and this only involve with one archwire rotation motion. Therefore, from the simplified view, we should try 
to avoid the gripper reverse processing in close loop bending process. We should adopt the processing mode of gripper forward bending to make the archwire rotate 90 degrees.

When orthodontics patient's dentition is overcrowded, opening vertical loop is used to open a large local clearance. When the two open vertical loop is used as a strength unit, it can rise and lower the twist of the lingual and lip direction. It is a kind of close loop used for orthodontic fixed appliance. Aiming to the shape character of open vertical loop, the process and archwire shape of each process of open large vertical bending is demonstrated, is as shown in Figure 4.
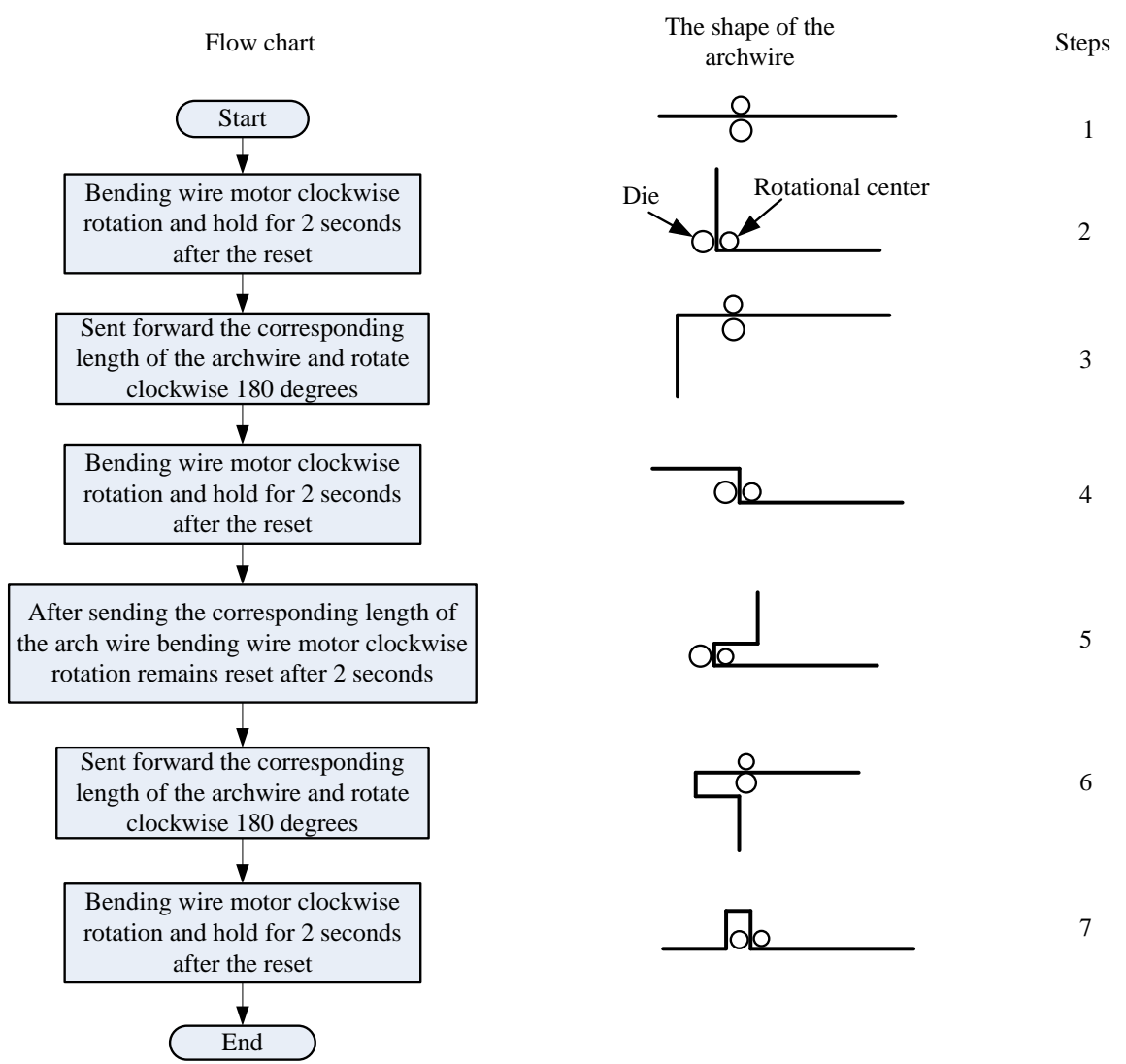

Figure 4. Bending Process of Open Vertical Loop

\subsection{Bending Process Scheme of T-shaped Loop}

T-shaped loop is relatively much more complicated than open vertical loop, and correspondingly the bending process is more. The bending process still can be realized by the cooperation of several movements. Main function of T-shaped loop is to close the gap generated with tooth extraction. Because its shape resembles with the shape of the english letter $\mathrm{T}$, hence be named as T-shaped loop. The use of the T-shaped loop increases the archwire length, and makes the orthodontic force softer and more durable. This avoids the moved forward disadvantages of teeth by excessive force. T-shaped loop bending process and the shape of the archwire is shown in Figure 5. 


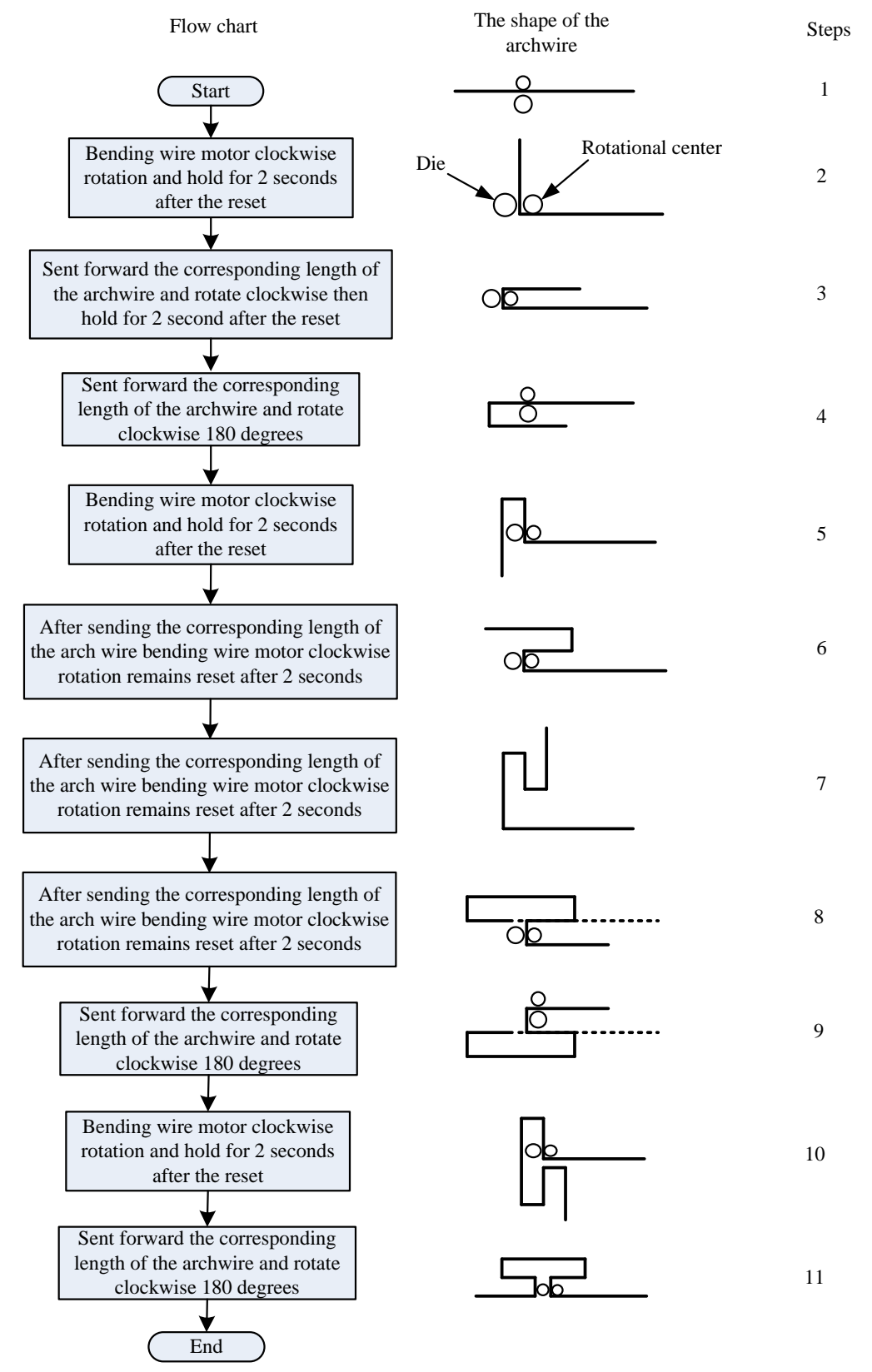

Figure 5. Bending Process Scheme of T-shaped Loop

\section{Structure Design of Orthodontic Archwire Bending Robot}

In the bending process, the moving distances of orthodontic archwire bending robot are relatively short, but the requirements for the moving accuracy is high. We select lead-screw mechanism as a moving carrier of the archwire bending robot, is shown in Figure 6. Prototype of archwire bending robot is as shown in Figure 7. Orthodontic archwire bending robot mechanism is composed of the base, the lead-screw drive platform, the archwire rotary motor, the supporting structure of archwire, bending die, cog belt drive mechanism and bending motor of archwire. The lead-screw drive platform as orthodontic archwire bending robot body, is responsible for supporting the whole robot system and transfer the movement. The fixture 
which install on shaft of the archwire rotary motor provide with clamping the orthodontic archwire and rotating the archwire to the specified angle. The supporting structure of archwire is used to maintain the fixed archwire in the archwire bending forming process. The cog belt drive mechanism drives the bending die rotation to realize the archwire bending.

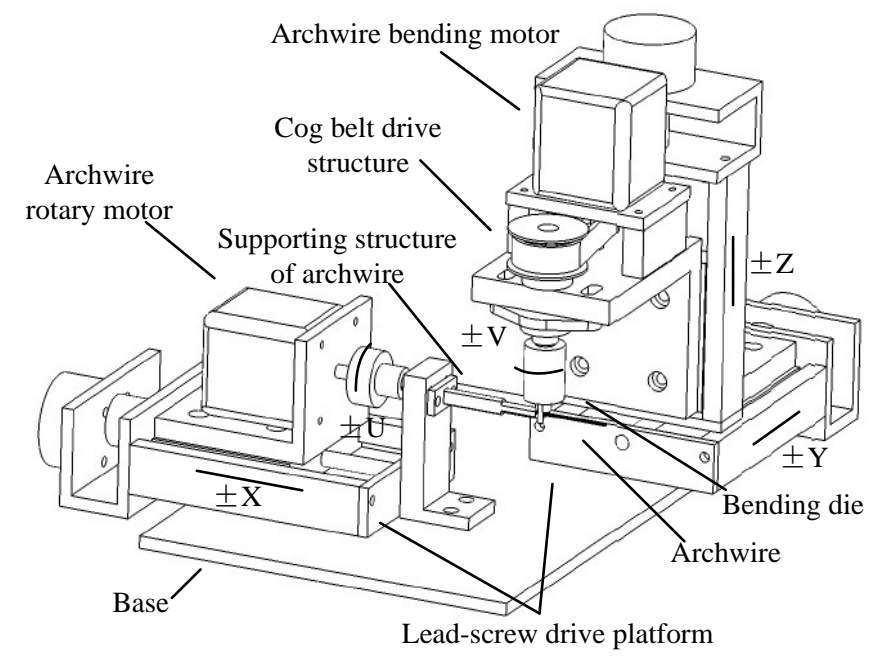

Figure 6. Mechanism Design of Archwire Bending Robot

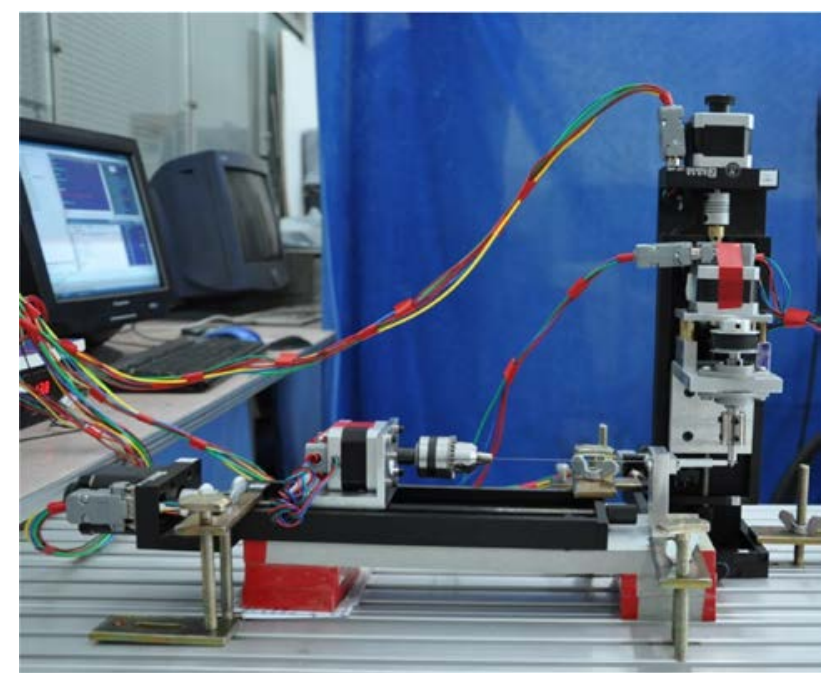

Figure 7. Prototype of Archwire Bending Robot

\section{Conclusion}

Archwire bending is conducted by orthodontist in orthodontic treatment. However, this procedure is boring and time-consuming. In order to reduce the dependence on human technology in archwire bending forming, decrease the labor intensity of dentists and technicians, and improve the degree of automation of the orthodontic process. This paper proposes to use robots to replace dentists for completing orthodontic wires bending. 
(1) Quantitative model of orthodontic wires with canine eminace is established based on Power function model.

(2) On the basis of bending process analysis of orthodontic wires, the overall structure, bend die and archwire supporting part of archwire bending robot for orthodontic treatment is designed.

\section{Acknowledgements}

We are extremely grateful to the anonymous referees for their most insightful and constructive comments, which have enabled us to improve the manuscript significantly. The authors are also grateful to the Editor in Chief and the technical editor for reviewing and editing the paper. This research were supported by the National Natural Science Foundation of China (Grant No. 51205093, 50675054) and the National High Technology Research and Development Program of China (Grant No. 2013AA0408).

\section{References}

[1] Z. YongDe and J. YuXiang, “The Control of Archwire Bending Robot Based on MOTOMAN UP6”, Proceedings of Biomedical Engineering and Informatics, Tianjin, China, (2009), pp. 1-5.

[2] J. Jingang and Z. Yongde, "Motion Planning and Synchronized Control of the Dental Arch Generator of the Tooth-arrangement Robot”, The International Journal of Medical Robotics and Computer Assisted Surgery, vol. 9, no. 1, (2013), pp. 94-102.

[3] J. Jingang and Z. G. Yongde, "Collaborative Simulation and Experimentation of Dental Arch Generator of Multi-manipulator Tooth-arrangement Rrobot”, International Journal of Advanced Robotic Systems, (2012).

[4] G. C. Burdea, S. M. Dunn and C. Immendorf, "Robotic System for Dental Substraction Radiography", Proceedings of the 1991 IEEE International Conference on Robotics and Automation, Sacramento, California, (1991), pp. 2056-2062.

[5] H. Takanobu, T. Yajima and M. Nakazawa, "Quantification of Masticatory Efficiency with a Mastication Robot”, Proceedings of the 1998 IEEE International Conference on Robotics and Automation, Leuven, Belgium, (1998), pp. 1635-1640.

[6] H. Takanobu, T. Maruyama and A. Takanishi, "Universal Dental Robot 6-DOF Mouth Opening and Closing Training Robot WY-5”, CISM-IFToMM Symposium on Theory and Practice of Robots and Manipulators, vol. 20, no. 7, (2000), pp. 33-34.

[7] T. Inoue, F. Yu and T. Nasu, "Development of a Clinical Jaw Movement Training Robot for Intermaxillary Traction Therapy”, Proceedings of 2004 IEEE International Conference on Robotics and Automation, New Orleans, LA, (2004), pp. 2492-2497.

[8] W. Birkfellner, K. Huber and A. Larson, "Modular Software System for Computer-aided Surgery and its First Application in Oral Implantology”, IEEE Transactions on Medical Imaging, vol. 19, no. 16, (2000), pp. 616-620.

[9] M. Alcaniz, F. Chinesta and C. Monserrat, "An Advanced System for the Simulation and Planning of Orthodontic Treatments”, Visualization in Biomedical Computing, vol. 1131, no. 5, (1996), pp. 511-520.

[10] L. Beldie, B. Walker and Y. Lu, "Finite Element Modelling of Maxillofacial Surgery and Facial Expressionsa Preliminary Study”, The International Journal of Medical Robotics and Computer Assisted Surgery, vol. 6, no. 1, (2010), pp. 422-430.

[11] Y. Zhang and J. Jiang, "Study on Precise Acceleration/deceleration Planning of Archwire Bending Robot", ICIC Express Letters, vol. 7, no. 1, (2013), pp. 73-78.

[12] Y. Zhang and J. Jiang, “Optimization Algorithm of Control Points Planning of Archwire Bending Forming”, International Journal of Digital Content Technology and its Applications, vol. 6, no. 22, (2012), pp. 590-599. 